\title{
ESSAY
}

\section{Engineering: Worldwide ebb}

\author{
In the last in our series on being human, Melanie Moses gets to grips with humanity's greatest challenge: \\ how to reduce the demand for energy in increasingly complex, networked and energy-dependent societies.
}

Humans consume resources equivalent to more than half the production achieved by all the plants and other primary producers on Earth. Our ability to do so, and to distribute those resources across the globe on a scale unparalleled in non-human systems, stems in part from infrastructure networks that connect us to each other and to our environment.

Engineered distribution networks, such as electric-power grids, oil pipelines, railroads, airports, trade routes and banking systems, and the communication networks that their coordination requires, provide the channels through which people, diseases, resources and ideas now move through the world. They determine who we meet, where we travel and how much we consume.

Many of our most pressing global challenges stem from flows over these networks. Air, rail and road networks vastly increase the likelihood of a pandemic by allowing billions to be infected by a virus at an unprecedented speed. Shipping networks carry energy from a few oil-rich locations to distant consumers, fuelling potentially catastrophic climate change. Even less tangible networks have tremendous impact on humanity: most economists failed to predict the speed and extent of the recent financial crisis partly because they didn't understand the nature of the networks through which it spread.

To manage our impact on the environment and understand the ramifications of our actions in an increasingly interconnected world, we need a macroscopic view as well as a detailed understanding of the structure of the networks we have created. The bigger picture is beginning to emerge from theoretical approaches that reveal the structure and dynamics of networks, how networks change as they grow, and how networks constrain individual behaviour.

\section{A question of size}

In the past decade, complex network theory has begun to describe the structural features of networks. It shows, for example, that in most social networks, many people have only a few connections whereas a small number of individuals are highly connected. The connections are also clustered: for example, we tend to know lots of our friends' and colleagues' acquaintances, but are less likely to know people from different professions or social groups. Understanding the structure of networks should certainly help reduce the spread of disease, or facilitate the spread of trust in a financial system. But it doesn't shed much light on how physical resources move through networks.

The Metabolic Theory of Ecology (MTE) offers one way to understand the dynamics of flow through networks. The mathematical foundation of MTE was developed a decade ago by a group of biologists and physicists who wanted to explain why so many characteristics of plants and animals systematically depend on their mass in a very peculiar way. The theory posits that much of the life history of an animal (such as how long it lives, how often it reproduces and how much it eats) is determined by geometric and dynamic properties of the cardiovascular network that controls its metabolism.

According to the theory, the larger the animal, the longer its cardiovascular system (its network of arteries and capillaries) takes to deliver resources to its cells. That delivery time, which in turn dictates the animal's metabolic rate, is proportional to the animal's mass raised to the power of $1 / 4$. Thus, because its circulatory system works less efficiently, an elephant grows systematically more slowly than a mouse, with a slower heart rate, a lower reproductive rate and a longer lifespan.

Biologists disagree over exactly how much slower the metabolism of larger animals is, and whether the networks-based explanation for the relationship between mass and metabolism is the correct one. However, the implications of this basic idea - that networks become predictably less efficient as they grow - are profound. Indeed, MTE offers insights that could revolutionize the way we understand, predict and manage large networked systems. As well as suggesting that larger systems process energy proportionally more slowly than smaller ones, it implies that the rate at which a system processes energy drives much of its broad-scale behaviour, whether that system is an organism, society or technology.

\section{A common trend}

Applying MTE to human social systems sheds light on the well-known but little understood decline in fertility rates that occurs with economic development. As societies consume more energy, people become wealthier but they also have fewer children. Today that energy primarily takes the form of fossil fuels. The average human uses up only about 100 watts from eating food, consistent with predictions based on body size. But in North America, each person uses an additional 10,000 watts from oil, gas, coal and a smattering of renewable sources, all of which are delivered through expansive, expensive infrastructure networks.

The decline in fertility rates with economic growth, called the demographic transition, has puzzled human-life-history theorists for decades: that the people with the most resources have the fewest offspring apparently contradicts basic Darwinian expectations, particularly as the gain in fitness resulting from improved offspring survival is far too small to compensate for the drop in birth rates. But MTE shows that this pattern is not unusual. In fact, across contemporary nations, the decline in human birth rates with increased energy consumption is quantitatively identical to the decline in fertility rate with increased metabolism in other mammals. Put another way, North Americans consume energy at a rate sufficient to sustain a 30,000-kilogram primate, and have offspring at the very slow rate predicted for a beast of this size.

Does the common pattern shared by humans and other mammals point to a common cause? In mammals, the amount invested in each offspring increases with the size of the animal, but, as discussed above, the proportion of energy available for reproduction declines as animals get bigger. Thus, an elephant takes longer (by a factor of mass raised to the power of $1 / 4$ ), than a mouse to acquire the resources needed to reproduce. It may be that humans similarly invest a constant fraction of available resources into each child, but take longer to acquire that fraction in wealthier societies. According to this hypothesis, as our infrastructure grows, we get more out of it, but we must invest proportionally more into it, reducing the energy and capital left to invest in the next generation. 


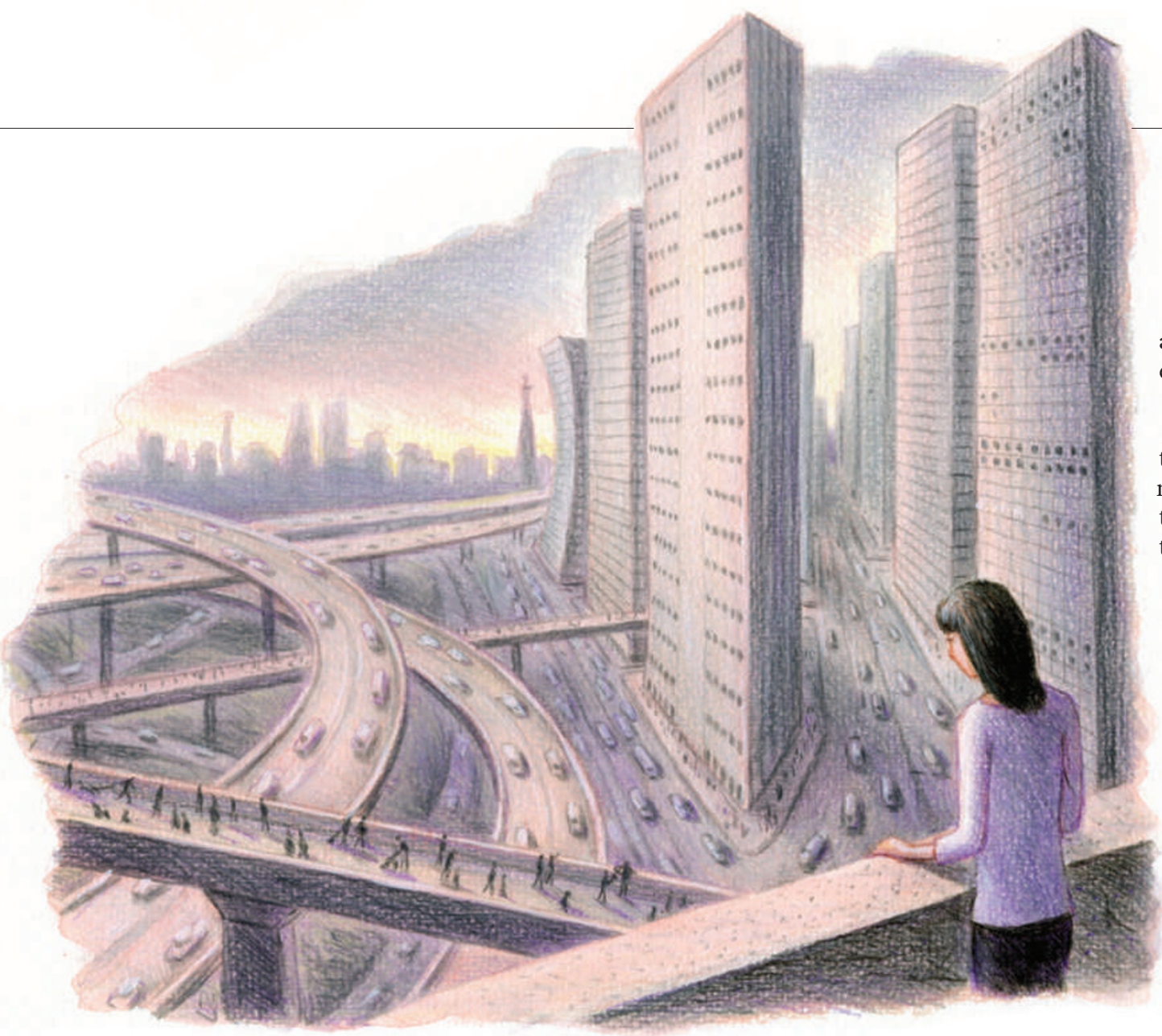

deliver energy less efficiently when they transport goods over longer distances. Global agricultural production increased six-fold from 1900 to 2000 by increasing energetic investment in agriculture 80 -fold. This appalling return on investment in the energy used to fertilize, harvest and transport food means that we now put more energy into acquiring food than we obtain from eating it. Better network designs are critical to ensure that the transportation networks, technologies and economies of the future give far better returns on energetic investment, even as they grow to serve more people. The world's remaining oil reserves are clustered in a few locations far from most consumers. But a more efficient, decentralized infrastructure could be built to deliver energy harvested from wind, solar and tidal resources.

Already, potentially more efficient ways to design infrastructure are emerging. My colleagues and I recently showed that some at least partially decentralized networks, such as computer networks and urban roads in cities (where half the world's population now resides), can

Alternative explanations for the demographic transition include cultural traditions that enable women to have fewer children than they are biologically capable of having, such as birth control or marrying late. But these mechanisms don't explain why women choose to delay having children in the first place. Another theory, based on 'embodied capital', proposes that as societies become wealthier, greater educational investments are made in each child to make them competitive in labour markets. This idea is broadly consistent with the hypothesis outlined above. Interestingly, years spent in education are strongly inversely correlated with fertility rates across nations.

In the face of climate change, the correlation between the decline in birth rates in wealthy nations and a sharp rise in energy consumption is alarming. Even the government-mandated fertility-rate reduction in China, which has resulted in a $70 \%$ drop in birth rate in three decades, has been accompanied by a dramatic increase in per capita energy consumption. If such correlations continue to hold, the projected 9 billion people alive in 2050 would achieve zero population growth only if each person consumed close to what the average European consumes today. That would increase total human consumption by ten times.

The recent economic crash demonstrates the fragility of financial networks and the tremendous impact their collapse can have; transportation networks facilitate the spread of disease; and distribution networks deliver sufficient energy to enable us to alter the planet's climate. Yet technological developments continue to connect more of the world's people. Moreover, achieving further economic growth, particularly in developing nations, will probably require more energy and therefore even more expansive networks.

\section{Efficiency drive}

Several crucial messages are emerging from early work on human-engineered networks. Human societies are complex systems that persist by consuming energy, but energy consumption cannot be lessened simply by reducing individual demand. Any one person's consumption and, possibly, fertility rate, is affected by structures at higher levels. Relating the behaviour of individuals to global-scale problems will require understanding those individuals as nodes in a network, in which the behaviour of one affects the whole society and where the collective behaviour of the society constrains the behaviour of individuals.

Another key message is that centralized networks, in which resources flow from a central place out to scattered destinations, increase in size more efficiently than purely centralized ones. For example, our models show that traffic, and so oil consumption, can be proportionally reduced as cities expand, as long as multiple recreational and commercial centres are placed near residential areas. Moreover, Luís Bettencourt and his colleagues recently showed that certain factors, such as innovation and wealth creation, increase superlinearly with city population. In this instance, the more people in a city, the more each person benefits from the collective ability to interact.

In the decades ahead, we need to understand how social and infrastructure networks constrain individual behaviour, and structure cities and societies in ways that increase innovationinducing interactions but reduce transport and travel distances. By doing so, we'll stand a better chance of meeting the needs of a large, voracious and growing human population without decimating the resources available to future generations.

Melanie Moses is at the Department of

Computer Science, University of New Mexico,

Albuquerque, NM 87131.

e-mail:melaniem@unm.edu

See http://tinyurl.com/d6ck5c for further reading. For more on Being Human, see www.nature.com/ nature/focus/beinghuman. 